\section{Comportamentos de risco à saúde de irmãos e sua influência nos adolescentes}

\section{Influence of health risk behaviors siblings on the adolescents}

\author{
Chirle 0 Raphaelli \\ Priscila M Nakamura² \\ Mario R Azevedo? \\ Pedro C Hallal ${ }^{1}$
}

\section{RESUMO}

Objetivou-se verificar a influência do comportamento de risco à saúde dos irmãos mais velhos e mais novos no comportamento dos adolescentes. Participaram do estudo 375 adolescentes de escolas municipais de Barão do Triunfo/RS e 175 irmãos. Foram avaliados os seguintes comportamentos de risco à saúde, de acordo com seus respectivos pontos de corte: inatividade física ( $<300$ minutos de atividades físicas semanais), fumo (uso de cigarros no último mês), consumo de álcool (bebida alcoólica no último mês) e excesso de peso (> percentil 95 do IMC), em adolescentes e irmãos (mais novos e mais velhos). Teste de qui quadrado e de regressão logística foram realizados. As prevalências de inatividade física, fumo, consumo de álcool e excesso de peso nos adolescentes foram de 47,9\%, 4,3\% 27\%, e $22,1 \%$, respectivamente. Adolescentes com irmãos mais novos fumantes apresentaram maior prevalência $(33,3 \%)$ do uso de cigarros $(\mathrm{p}=0,01)$ comparados com adolescentes com irmãos sem esse comportamento. A odds ratio do adolescente fumar tendo irmão mais novo fumante foi de 16,5. O consumo de bebida alcoólica pelo irmão, mais velho ou mais novo, teve associação com consumo pelo adolescente $(\mathrm{p}=0,04 \mathrm{e} \mathrm{p}=0,03)$. A chance desse consumir álcool quando seu irmão consumiu foi de 3,4 vezes, dentre os mais novos e de 3,0 vezes dentre os mais velhos. O fumo e o consumo de álcool do irmão estão associados aos mesmos comportamentos dos adolescentes. Desse modo, há necessidade de direcionar ações de promoção da saúde abrangendo adolescentes e seus irmãos.

\section{PALAVRAS-CHAVE}

Adolescente; Irmãos; Comportamento de risco; Saúde.

\footnotetext{
ABSTRACT

This study aimed to investigate the influence of risk behavior to the health of older and younger brothers in the behavior of adolescents. The study included 375 adolescents from public schools of Barão of Triunfo/RS and 175 brothers. We evaluated the following health risk behaviors, according to their respective cut-off points: physical inactivity (<300 minutes of weekly physical activity), tobacco (cigarette use in the past month), alcohol consumption (alcohol in the last month) and overweight (> 95th percentile BMI) in adolescents and siblings (younger and older). Chi-square test and logistic regression were performed. The prevalence of physical inactivity, smoking, alcohol consumption and overweight adolescents were $47.9 \%, 4.3 \%, 27 \%$ and $22.1 \%$. Adolescents with younger siblings smokers had a higher prevalence (33.3\%) of smoking $(p=0.01)$ compared with adolescents with siblings without this behavior. The odds ratio adolescent smoking with younger brother smokers was 16.5. The consumption of alcohol by brother, older or younger, was associated with consumption by adolescents $(p=0.04$ and $p=0.03)$. The chance of consuming alcohol when bis brother consumed was 3.4 times, among the newest and 3.0 times among the elderly. The smoke and the brother alcohol consumption are associated with the same behaviors of adolescents. Thus, there is need to direct bealth promotion activities involving teenagers and their siblings.
}

\section{KEYWORDS}

Adolescent; Siblings; Risk behavior; Health.
Rev Bras Ativ Fis Saúde p. 744-754 DOI

http://dx.doi.org/10.12820/rbafs.v.19n6p744

1 Universidade Federal de Pelotas, Pelotas. Programa de Pós-Graduação em Educação Física, Pelotas-RS-Brasil.

2 Universidade Estadual Paulista -Programa de Pós-Graduação em Ciências da Motricidade Campus Rio Claro-SP e Instituto Federal de Educacão, Ciência e Tecnologia - Sul de Minas Gerais. Câmpus Muzambinho-MG, Brasil. 


\section{INTRODUÇÃO}

Os adolescentes constituem-se como um grupo prioritário para a promoção de saúde, pois é nessa fase que eles inicialmente se expõem a diversas situações de risco, as quais podem contribuir para o surgimento de doenças e agravos não transmissíveis na vida adulta ${ }^{1}$. Essas situações de risco, incluem o consumo de drogas, a alimentação inadequada, o sedentarismo e o sexo não protegido, e podem ser influenciadas por estímulos ambientais e familiares no qual $\mathrm{o}$ adolescente está inserido.

O adolescente perpassa uma fase marcada por mudanças psicológicas, físicas e psicossociais com maior ganho de autonomia na vida podendo implicar na exposição aos riscos à saúde com desfechos desfavoráveis imediatos ou tardios na vida, como exemplo, o uso do tabaco e do álcool ${ }^{1}$.

A família é o primeiro e mais importante ambiente social da criança e do adolescente e é fundamental na formação de um estilo de vida saudáveis. As tradições de família e a influência de pares, amigos e irmãos podem ser importantes definidores de atitudes, crenças e circunstâncias nas escolhas e atitudes relativos a comportamentos saudáveis ou não saudáveis. Identificar esses estímulos é de extrema importância devido ao aumento da prevalência do uso de cigarros, consumo abusivo de bebidas alcoólicas, inatividade física e o excesso de peso entre os adolescentes ${ }^{2-6}$. A composição do núcleo familiar que o adolescente está inserido pode influenciar comportamentos saudáveis ou de risco à saúde, por exemplo, adolescentes com irmãos fumantes na família apresentam risco mais elevado de experimentar cigarros ${ }^{2}$.

Nesse sentido, os comportamentos de risco à saúde, geralmente, refletem o contexto social, demográfico e cultural de onde o jovem está inserido. Pesquisadores têm investigado o ambiente familiar e consideram que, pais, mães e irmãos, possam servir como modelo, tanto positivo como negativo, aos comportamentos adotados na infância e na adolescência ${ }^{7,8}$. Todavia, a influência do irmão sobre o comportamento do adolescente parece ser mais importante do que a influência dos pais e amigos para adoção de maus hábitos de comportamento, pois passam mais tempo juntos, convivem por toda a vida e há uma relação psicossocial muito forte 9 . Todavia, pouco se sabe se o comportamento do irmão mais velho influência mais o comportamento do adolescente quando comparado com a influência do irmão mais novo.

Ademais, diversos estudos identificam comportamentos de saúde ou de risco à essa em jovens residentes da zona urbana ${ }^{2,3}$, porém, a literatura é escassa de dados de moradores de zona rural e em municípios pequenos. Com isso, o objetivo do presente estudo foi verificar a associação de comportamentos de risco à saúde (inatividade física, fumo, consumo de álcool e excesso de peso) dos irmãos mais velhos e mais novos com os dos adolescentes em um município de zona rural do Rio Grande do Sul. Os resultados do presente estudo poderão ajudar a elucidar a influência dos irmãos na adoção de maus hábitos de comportamento e dessa forma direcionar intervenções que foquem tanto nos adolescentes como em seus irmãos.

\section{MÉTODOS}

Estudo transversal foi realizado em Barão do Triunfo, RS, município, com ter- 
ritório de 436,4 $\mathrm{km}^{2}$, tem 7.018 habitantes, sendo que $90 \%$ residem na zona rural. A economia local se baseia na fumicultura e na bovinocultura. $\mathrm{O}$ município contem dez escolas, sendo oito municipais distribuídas na zona rural, uma escola estadual e uma instituição filantrópica localizadas na zona urbana. As municipais atendem a pré-escola até o ensino fundamental, a estadual, o ensino médio, e a instituição filantrópica, escolares portadores de deficiências. Ainda assim, a prevalência de analfabetismo, de acordo com o último Censo é de $9,8 \%$ na população com 15 anos ou mais ${ }^{10}$.

O presente estudo foi aprovado pelo Comitê de Ética em Pesquisa com seres humanos da Escola Superior de Educação Física da Universidade Federal de Pelotas, RS, sob o protocolo 068/2009. Os responsáveis pelos adolescentes assinaram o Termo de Consentimento Livre e Esclarecido e aos jovens garantiu-se, em todas as fases da pesquisa, o anonimato, a liberdade de recusa e a possibilidade de retirada do consentimento.

\section{Participantes}

Em 2009 haviam 913 alunos matriculados no Ensino Fundamental da Rede Municipal de Educação do referido município, e, desses, 412 eram alunos de $5^{\circ}$ a $8^{\circ}$ anos, distribuídos em três escolas de ensino fundamental completo ${ }^{11}$. O presente estudo foi realizado entre março e setembro de 2010 incluindo todos os adolescentes matriculados entre o $5^{\circ}$ e $8^{\circ}$ anos, bem como seus irmãos (biológicos ou não) com idade entre 10 e 19 anos e moradores da mesma residência ou na mesma propriedade rural do adolescente. Excluíram-se do estudo, indivíduos com incapacidade mental de responder ao questionário.

\section{Procedimentos}

Antes do início da coleta de dados, o gestor da Secretaria Municipal de Educação e os diretores das escolas foram contatados para esclarecimento e permissão à execução da pesquisa. Também, previamente à coleta, foi realizado o estudo piloto com adolescentes semelhantes a amostra estudada residentes em um município vizinho com intuito de verificar a qualidade de entendimento do questionário.

Dentre as escolas municipais, as que continham escolares com idades elegidas para o estudo, concordaram em participar da pesquisa, totalizando três escolas participantes.

No primeiro momento, foram efetuadas, pelo menos, três visitas em cada sala de aula para aplicar o questionário de comportamento de risco à saúde (inatividade física, fumo e consumo de álcool) e em sala reservada a aferição das medidas antropométricas (peso e estatura) nos adolescentes, bem como, em seus irmãos (velhos e/ou novos) que estudavam nas mesmas escolas.

Para o presente estudo foi definido como irmão mais novo do adolescente, aquele com idade mais próxima a idade do adolescente, independente do número de irmãos mais novos da família. Para o irmão mais velho foi definido como sendo com a idade mais próxima do adolescente, independente do número de irmãos mais velhos da família. A inclusão de, no máximo, um irmão mais velho e um mais novo de cada adolescente, nos dados do estudo aconteceu para possibilitar a logística e o trabalho de campo. Em virtude dos mesmos ocorrerem em zona rural a coleta de dados de irmãos não matriculados nos domicílios poderia inviabilizar o estudo pela morosidade até localizar os irmãos e acessar suas residências. 
Na segunda etapa, foram obtidos dados dos irmãos mais velhos e mais novos que não estudavam nas mesmas escolas dos adolescentes, sendo que para isso foi necessário realizar uma triagem nas outras escolas da rede municipal para localizar os irmãos e, em seguida, agendar a coleta de dados. Os irmãos que não estavam matriculados na rede de ensino foram chamados nas três escolas onde os adolescentes estudavam para a obtenção das informações necessárias. Quando os irmãos não compareceram na data agendada foi remarcada outra data e/ou após foi realizada no próprio domicílio do irmão.

Todas as entrevistas com os adolescentes e seus irmãos foram realizadas pela pesquisadora do presente artigo. As entrevistas foram realizadas de forma assistida, sendo que a pesquisadora explicou uma questão por vez e os próprios adolescentes/irmãos responderam as questões. O preenchimento do questionário pelos adolescentes durou em média 40 minutos.

\section{Medidas antropométricas}

As medidas antropométricas foram coletadas por três professores de educação física, previamente treinados por oito horas. O controle de qualidade foi realizado em $10 \%$ das medidas antropométricas dos adolescentes para a correção dos erros e conferência dos dados coletados. Em local reservado, medidas de massa corporal e estatura foram obtidas dos adolescentes e irmãos, onde os mesmos portavam roupas leves, sem calçados e em posição ortostática ${ }^{12}$ através da balança digital marca PLENNA (capacidade de $150 \mathrm{~kg}$ e precisão de $100 \mathrm{~g}$ ). Para as medidas de estatura, foi utilizado o estadiômetro tipo portátil da marca SANNY (medida até $210 \mathrm{~cm}$ e precisão de $1 \mathrm{~mm}$ ).

Para verificação do excesso de peso, considerado um comportamento de risco à saúde, calculou-se o estado nutricional a partir do índice de massa corporal (IMC) e o mesmo foi classificado em percentis segundo idade e sexo ${ }^{13}$. Os adolescentes com IMC acima do percentil 95 foram classificados como expostos à esse comportamento de risco.

\section{Questionários}

A inatividade física, foi avaliada a partir de questionário proposto por Bastos et al. (2008) $)^{3}$ o qual investiga as atividades realizadas nos sete dias anteriores à aplicação do questionário, excluindo as atividades realizadas durante as aulas de educação física escolar. Esse instrumento coleta informações da frequência e duração de cada atividade realizada. O ponto de corte utilizado foi de 300 minutos por semana de atividades físicas, incluindo as praticadas no lazer e no deslocamento, sendo que os adolescentes e seus irmãos que não atingiam esse ponto de corte, foram considerados como tendo esse comportamento de risco à saúde, inatividade física ${ }^{14}$.

Para avaliar o consumo de álcool e fumo, foram utilizadas questões propostas pelo Centro Brasileiro de Informações Sobre Drogas Psicotrópicas ${ }^{15}$. Foram considerados como expostos a esses comportamentos os adolescentes e os irmãos que referiram ter consumido qualquer quantidade de bebida alcoólica ou ter usado qualquer quantidade de cigarros no último mês ${ }^{15}$.

\section{Análise estatística}

A análise consistiu na obtenção da existência de relação entre uma variável dependente, relacionada ao adolescente (variável dependente/resposta) e uma 
independente, relativa ao irmão (variável explicativa) (mais novo ou mais velho). Para verificar essas associações foram realizados testes do Qui quadrado de regressão logística, com nível de significância de $\mathrm{p}<0,05$ realizados no programa STATA 11.0. Cabe destacar que a medida de efeito utilizada foi odds ratio que pode superestimar a razão de prevalência quando o desfecho utilizado é muito comum o que não aconteceu no presente estudo, com exceção do comportamento de risco excesso de peso.

\section{RESULTADOS}

Dentre os 412 escolares elegíveis para o estudo, 375 adolescentes aceitaram participar do estudo, sendo 53\% meninos. Desses, 175 adolescentes $(51 \%$ meninas) tinham irmãos mais novos ( $\mathrm{n}=77$ ) (52\% meninas) e/ou irmãos mais velhos ( $\mathrm{n}=97)$ (52\% meninos). As médias de idade foram de 12 anos $(\mathrm{DP} \pm 1,8)$, 15 anos $(\mathrm{DP} \pm 1,8)$ e 11 anos $(\mathrm{DP} \pm 1,4)$ para os adolescentes, os irmãos mais velhos e os mais novos, respectivamente. Dentre os irmãos, $58 \%$ dos mais velhos apresentaram 3 ou mais anos de idade que o adolescente e $38 \%$ dos mais novos apresentaram 3 anos ou menos anos de idade comparados com o adolescente.

Dentre os adolescentes com irmãos, $47,9 \%$ eram inativos, sendo que $55,2 \%$ e 55,4\% dos irmãos mais velhos e mais novos, respectivamente. A prevalência de uso de cigarros e de consumo de álcool dos adolescentes foi de 4,3\% e $27,3 \%$, respectivamente. Os irmãos mais velhos apresentaram prevalências de fumo e de consumo de álcool, de 7,7\% e de 36,3\% respectivamente. Já os irmãos mais novos apresentaram uso de $4,0 \%$ de fumo e $17,1 \%$ de álcool. O excesso de peso, nos irmãos mais novos apresentou prevalência de 37,7\% dos irmãos mais velhos, 25,5\% e nos adolescentes de 22,1\% (Tabela 1 ).

TABELA 1 - Comportamentos de risco à saúde em adolescentes, seus irmãos mais velhos e irmãos mais novos. Barão do Triunfo, RS. 2010.

\begin{tabular}{|c|c|c|c|}
\hline \multirow{2}{*}{ Comportamentos de risco à saúde } & Adolescentes $^{\mathrm{a}}$ & Irmãos mais velhos & \multirow{2}{*}{$\begin{array}{c}\text { Irmãos mais novos } \\
\% \mathrm{n}\end{array}$} \\
\hline & $\% \quad n$ & $\% n$ & \\
\hline $\begin{array}{l}\text { Atividade física insuficiente } \\
<300 \mathrm{~min} / \mathrm{sem}\end{array}$ & $47,9(142)$ & $55,2(87)$ & $55,4(74)$ \\
\hline $\begin{array}{l}\text { Fumo } \\
\text { Qualquer quantidade de cigarros } \\
\text { nos últimos } 30 \text { dias. }\end{array}$ & $4,3(140)$ & $7,7(91)$ & $4,0(75)$ \\
\hline $\begin{array}{l}\text { Consumo de Álcool } \\
\text { Qualquer quantidade de bebida } \\
\text { alcoólica nos últimos } 30 \text { dias. }\end{array}$ & $27(148)$ & $36,3(91)$ & $17,1(76)$ \\
\hline $\begin{array}{l}\text { Excesso de peso } \\
\text { Classificado pelo IMC }\end{array}$ & $22,1(154)$ & 25,5 (98) & $37,7(77)$ \\
\hline
\end{tabular}

andivíduos que possuem irmão mais novo ou mais velho; bاMC: Índice de Massa Corporal classificado, segundo idade e sexo e classificado como excesso de peso o ponto de corte acima do percentil $95^{13}$.

A Tabela 2 demonstra a análise de comportamentos de risco à saúde dos adolescentes associados com os mesmos comportamentos dos irmãos mais novos. Jovens cujos irmãos mais novos são inativos apresentaram $51,2 \%$ de prevalência de inatividade física $(\mathrm{p}=0,07)$. Adolescentes que têm irmãos mais novos fumantes e que consomem bebidas alcoólicas apresentam, respectivamente, $33,3 \%$ de prevalência de fumo $(\mathrm{p}=0,01)$ e $40,0 \%$ de consumo de bebi- 
das alcoólicas $(\mathrm{p}=0,04)$. Irmãos mais velhos inativos, fumantes, consumidores de bebidas alcoólicas e com excesso de peso, apresentaram 60,4\% de adolescentes inativos $(\mathrm{p}=0,07), 16,7 \%$ fumantes $(\mathrm{p}=0,06), 33,3 \%$ consumidores de bebidas alcoólicas no último mês à entrevista $(\mathrm{p}=0,03)$ e $36 \%$ de adolescentes com excesso de peso $(\mathrm{p}=0,06)$.

TABELA 2 - Associação entre os comportamentos de risco à saúde dos irmãos mais novos e mais velhos com os mesmos comportamentos do adolescente. Barão do Triunfo, RS. 2010.

\begin{tabular}{|c|c|c|c|c|}
\hline \multirow[b]{2}{*}{$\begin{array}{l}\text { Variáveis relacionadas ao } \\
\text { irmão }\end{array}$} & \multicolumn{2}{|c|}{ Irmãos mais novos } & \multicolumn{2}{|c|}{ Irmãos mais velhos } \\
\hline & $\begin{array}{l}\text { \% da variável } \\
\text { correspondente ao } \\
\text { adolescente }\end{array}$ & Valor-pa & $\begin{array}{l}\text { \% da variável } \\
\text { correspondente ao } \\
\text { adolescente }\end{array}$ & Valor-pa \\
\hline Atividade Física insuficiente $(n=87)$ & & 0,07 & & 0,07 \\
\hline Não & 30,0 & & 41,0 & \\
\hline Sim & 51,2 & & 60,4 & \\
\hline Fumo $(n=87)$ & & 0,01 & & 0,06 \\
\hline Não & 2,9 & & 2,5 & \\
\hline Sim & 33,3 & & 16,7 & \\
\hline Consumo de álcool (n=89) & & 0,04 & & 0,03 \\
\hline Não & 23,1 & & 14,3 & \\
\hline Sim & 40,0 & & 33,3 & \\
\hline Excesso de peso $(n=98)$ & & 0,16 & & 0,06 \\
\hline Não & 14,6 & & 17,8 & \\
\hline Sim & 27,6 & & 36,0 & \\
\hline
\end{tabular}

aTeste do Qui-quadrado para heterogeneidade.

Quando a análise foi realizada com irmãos do mesmo sexo ( $\mathrm{n}=40)$, irmãos mais novos inativos, que fumavam, consumiam álcool e tinham excesso de peso tinham mais risco de ter adolescentes inativos (80,0\%), que fumavam $(33,3 \%)$, consumiam álcool $(36,4 \%)$ e apresentavam excesso de peso $(66,7 \%)$.

Quando a análise foi realizada com irmãos do mesmo sexo $(n=40)$, os irmãos mais novos inativos, que fumavam, consumiam álcool e tinham excesso de peso tiveram associação com os mesmos comportamentos dos adolescentes: inativos $(80,0 \%)$, que fumavam $(33,3 \%)$, consumiam álcool $(36,4 \%)$ e apresentavam excesso de peso (66,7\%) (dados não apresentados nas tabelas). Ainda com irmãos do mesmo sexo $(n=43)$, porém, com os mais velhos que eram inativos e com excesso de peso se associaram a inatividade física $(63,6 \%)$ e ao excesso de peso no adolescente $(50,0 \%)$ (dados não apresentados nas tabelas).

A tabela 3 demonstra dados de relação dos comportamentos de risco à saúde dos adolescentes relacionados aos mesmos comportamentos dos irmãos, mais novos ou mais velhos. A odds ratio para fumo nos adolescentes com irmãos mais novos fumantes foi de 16,5, porém, com significância estatística limítrofe. A chance dos adolescentes consumirem bebida alcoólica quando seus irmãos mais novos também consumiram foi de 3,4 vezes, com significância estatística no limite superior, e quando seus irmãos mais velhos consumiram foi de 3,0 vezes. Na análise, para o comportamento excesso de peso não houve relação entre as respostas dos irmãos e dos adolescentes, pois, os resultados da regressão não variaram. 
TABELA 3 - Comportamentos de risco à saúde do adolescente relacionados aos mesmos comportamentos do irmão mais novo ou mais velho, conforme regressão logística. Barão do Triunfo, RS. 2010.

\begin{tabular}{lcccc}
\hline \multicolumn{1}{c}{$\begin{array}{c}\text { Variáveis relacionadas } \\
\text { aos irmãos }\end{array}$} & $\mathrm{n}$ & $\mathrm{OR}$ & IC $95 \%^{\mathrm{a}}$ & Valor $-\mathrm{p}^{\mathrm{b}}$ \\
\cline { 3 - 5 } & & & & \\
\hline Atividade Física insuficiente & 74 & 2,4 & $0,9-6,3$ & 0,07 \\
\hline Irmãos mais novos & 87 & 2,2 & $0,9-5,2$ & 0,07 \\
\hline Irmãos mais velhos & & & $1,0-266,5$ & 0,05 \\
\hline Fumo & 71 & 16,5 & 0,11 \\
\hline Irmãos mais novos & 87 & 7,9 & $0,6-102,7$ & \\
\hline Irmãos mais velhos & & & & 0,05 \\
\hline Consumo de álcool & 75 & 3,4 & $0,97-11,6$ & 0,03 \\
\hline Irmãos mais novos & 89 & 3,0 & $1,06-8,5$ & \\
\hline Irmãos mais velhos & & & & \\
\hline
\end{tabular}

aR: odds ratio; ' ${ }^{\mathrm{D}} \mathrm{C}$ 95\%: Intervalo de confiança de $95 \%$.

\section{DISCUSSÃO}

O presente estudo investigou a associação de comportamentos de risco à saúde entre irmãos mais velhos e irmãos mais novos no comportamento dos adolescentes. Os principais resultados foram que adolescentes com irmãos mais novos que são inativos, fumam e consomem bebidas alcoólicas apresentaram maior probabilidade de serem fumantes, inativos e consumidores de bebidas alcoólicas ao serem comparados com os adolescentes cujos irmãos mais novos não possuíam esses comportamentos. Além disso, adolescentes com irmãos mais velhos que são inativos, fumam, ingerem bebidas alcoólicas e possuem excesso de peso, apresentaram adolescentes com maior prevalência desses comportamentos quando comparados com irmãos mais velhos sem esse comportamento.

$\mathrm{Na}$ amostra estudada, praticamente a metade dos adolescentes $(47,9 \%)$ e mais da metade dos irmãos mais velhos $(55,2 \%)$ e mais novos $(55,4 \%)$ não realizam 300 minutos por semana de atividades físicas. Essas frequências foram similares a prevalência de $56,9 \%$ verificado em adolescentes de todas as capitais brasileiras e no Distrito Federal ${ }^{6}$.

No presente estudo verificou-se que adolescentes com irmãos mais novos inativos apresentaram $67,7 \%$ maior risco de serem inativos em comparação com adolescentes cujos irmãos são ativos. De acordo com o estudo de coorte desenvolvido por Jose et al $(2011)^{16}$, foi verificado que meninas que tinham irmãs mais novas apresentaram menos chance de serem ativas na fase adulta e que há poucos estudos que verificaram a influência do irmão mais novo no comportamento do irmão mais velho. Dados do referido estudo demostra que a presença de irmão mais novo pode influenciar a prática de atividade física, pois, muitas vezes, os mais velhos são responsáveis em cuidar dos seus irmãos mais novos não restando tempo para a prática de atividade física ${ }^{16}$.

O mesmo aconteceu com a análise dos irmãos mais velhos $(60,4 \%)$, sendo que os adolescentes tiveram mais chance de serem inativos quando o seu irmão mais velho também era inativo. Dados semelhantes, mas referente ao comportamento positivo de prática de atividade física, foram apresentados em estudo de Seabra et al. (2008) ${ }^{17}$, quando mais de $72 \%$ dos adolescentes portugueses participaram de esportes quando os seus irmãos também participaram, 
encontrando associação significativa entre as variáveis, mas, sem verificar a ordem de idade dos familiares.

A prevalência de uso de cigarros dos irmãos mais velhos foi de $7,7 \%$, dos adolescentes de 4,3\% e dos irmãos mais novos foi de 4,0\%. Valores similares foram demonstrados nas capitais do país $(6,3 \%)^{2}$ e superiores foram encontrados na região sul: Florianópolis/SC (10,7\%), Curitiba/PR (12,6\%) e Porto Alegre/RS $(17,7 \%)^{18}$. Percebe-se uma tendência positiva entre a frequência de uso de cigarros e a idade dos jovens, o que é confirmado por alguns autores ${ }^{2,19}$.

$\mathrm{Na}$ análise de associação, adolescentes com irmãos mais novos fumantes eram mais prováveis de fumar em comparação com os adolescentes com irmãos não fumantes. $\mathrm{O}$ mesmo aconteceu na análise realizada entre irmãos mais velhos e os adolescentes. Confirmando nossos achados, a pesquisa de Abreu et al $(2011)^{19}$, realizada com jovens de 15 a 24 anos, mostrou que entre os adolescentes que fumam, $21,1 \%$ dos irmãos possuem o mesmo hábito. Alguns pesquisadores também encontraram a relação de modelo a esse comportamento entre irmãos mais velhos e mais novos ${ }^{20,21}$. Destaca-se, por exemplo, jovens que viram seus irmãos fumando em alguma ocasião possuem mais chance de usar cigarros que jovens que nunca viram ${ }^{22}$.

Autores nacionais têm investigado o consumo de bebidas alcoólicas realizados por adolescentes com base nos 30 dias anteriores à investigação ${ }^{23}$. No presente estudo, a prevalência de uso de álcool, com base nesse ponto de corte, foi maior nos adolescentes mais velhos e menor nos mais jovens, tendência confirmada por outros autores ${ }^{24}$. A prevalência apresentada pelos irmãos mais velhos $(36,3 \%)$ foi inferior ao estudo de Vieira et al. $(2008)^{23}$, a frequência apresentada pelos adolescentes (27,3\%) se mostrou similar ao estudo de Andrade et al. (2012) $)^{24}$ e por fim, a prevalência demonstrada pelos irmãos mais novos $(17,1 \%)$ foi superior aos dados do estudo de Strauch et al. $(2009)^{25}$.

O álcool é uma das drogas psicotrópicas que tem seu consumo admitido e incentivado pela sociedade e inúmeras vezes isso acontece dentro do próprio domicílio. É comum os jovens acessarem a bebida na sua própria residência ${ }^{26}$ e geralmente os irmãos mais velhos fazem a compra ou oferecem a bebida alcoólica aos mais novos ${ }^{27}$. Isso está de acordo com os resultados obtidos no presente estudo que o consumo de bebidas alcoólicas apresentado pelos jovens foi mais provável de se verificar em adolescentes que possuíam irmãos mais velhos e mais novos que consumiram álcool no último mês em comparação com os adolescentes que tinham irmãos que não consumiam bebidas alcoólicas. Isso mostra que o exemplo de consumo pelos irmãos pode servir de influência ao mesmo comportamento. Reforçando esses resultados, uma pesquisa mostra que os jovens são $71 \%$ mais propensos a usarem bebidas alcoólicas quando seu irmão mais velho fez uso de álcool comparados com adolescentes que seu irmão mais velho não fez uso de álcool ${ }^{20}$. Da mesma forma, estudo com escolares espanhóis demonstrou que ter irmãos consumidores de bebida alcoólica é fator de risco para o consumo, seja habitual ou esporádico, de álcool pelos adolescentes de 12 a 19 anos, sendo que os irmãos e irmãs consumidores apareceram como fator de risco anterior ao pai e a mã $\mathrm{e}^{28}$.

O excesso de peso, que atualmente é um problema de saúde pública em adultos, recentemente tem acometido a infância e a adolescência instigando, assim, o interesse de pesquisadores da área. Dados nacionais da Pesquisa $\mathrm{Na}$ cional de Saúde do Escolar PeNSE, divulgados pelo Ministério da Saúde, de- 
monstram a atenção tida com a saúde do adolescente brasileiro, mas, em contraponto revela $30,3 \%$ jovens escolares de nono ano do ensino fundamental estão acima do peso $^{29}$. Dados do presente estudo mostram que as prevalências de excesso de peso nos irmãos mais novos $(37,7 \%)$, mais velhos $(25,5 \%)$ e nos adolescentes $(22,1 \%)$ são próximas aos dados nacionais da referida pesquisa. No presente estudo, verificou-se que adolescentes cujos irmãos mais velhos possuíam excesso de peso tinham mais risco de ter sobrepeso ou obesidade quando comparados com irmãos eutróficos. Uma pesquisa, realizada com escolares de 6 a 10 anos de Kuwait, demonstrou que possuir um ou mais irmãos obesos está associado com maior risco de sobrepeso e obesidade do que não possuir irmãos com excesso de peso ${ }^{30}$. Nesse sentido, mudanças comportamentais são necessárias para a redução do excesso de peso nos adolescentes, assim como mudanças positivas que envolvam toda a família, especialmente entre irmãos para que se consiga êxito na redução de exposição aos riscos à saúde.

$\mathrm{Na}$ amostra estudada, as análises indicam chance de o adolescente fumar ou consumir bebida de álcool quando seu irmão mais novo também faziam esses comportamentos, mas, no limite da significância estatística. A chance do adolescente consumir álcool quando seu irmão mais velho consumiu foi de 3,0 vezes. Autores demonstram que adolescentes com irmãos mais velhos fumantes apresentam razão de odds maior que 2 vezes para o adolescente ser fumante 5 .

Cabe resgatar, que no presente estudo algumas limitações devem ser consideradas. Primeiro, por ter um delineamento transversal, a coleta dos desfechos e das variáveis de exposição se deu em um único momento, o que impede a avaliação de temporalidade nas associações encontradas. Outra limitação refere-se ao fato de que os dados do atual estudo foram coletados em Barão do Triunfo, RS, e isso sugere cautela na generalização dos resultados para a população em geral. Na coleta de dados foram obtidas informações de irmãos com idade mais próxima a do adolescente, apenas um indivíduo mais velho e um mais novo, isso foi o ponto decisivo para viabilizar a logística de campo e evitar perdas, pois, o ideal seria a coleta com todos os irmãos do adolescente o que ampliaria o trabalho de campo. Entretanto, um fator que garante confiabilidade dos resultados é que os dados foram coletados diretamente de cada um dos adolescentes e de seus irmãos, ou seja, não foram utilizados dados secundários de relato dos adolescentes para obter a informação do irmão, o que é realizado por alguns autores?

Os resultados do presente estudo permitem concluir que há uma elevada proporção de adolescentes com níveis insuficientes de atividade física, uso de cigarros, consumo de álcool e com excesso de peso e com irmãos que possuem os mesmos comportamentos.

Os comportamentos de risco à saúde dos irmãos (fumo e consumo de álcool) estão associados aos mesmos comportamentos dos adolescentes, especialmente de irmãos mais velhos sendo modelo positivo no consumo de bebida alcoólica. A exposição simultânea a comportamentos de risco à saúde, tanto pelos jovens como seus irmãos, pressupõe que o ambiente familiar pode contribuir para as escolhas positivas ou negativas e isso possibilita aos adolescentes imitar a mesma atitude de seus irmãos. Finalmente, os dados aqui apresentados podem contribuir e direcionar as ações e programas de promoção da saúde para o ambiente familiar, abrangendo especialmente os adolescentes e seus irmãos. 


\section{Agradecimentos}

À Prefeitura Municipal de Barão do Triunfo, RS, aos docentes das escolas municipais que auxiliaram de alguma forma na realização da pesquisa e aos escolares e seus irmãos que participaram da coleta de dados.

\section{REFERÊNCIAS}

1. Malta DC, Sardinha LMV, Mendes I, Barreto SM, Giatti L, Castro IRR, et al. Prevalência de fatores de risco e proteção de doenças crônicas não transmissíveis em adolescentes: resultados da Pesquisa Nacional de Saúde do Escolar (PeNSE), Brasil, 2009. Ciênc. Saúde Coletiva. 2010; 15(2): 3009-19.

2. Barreto SM, Giatti L, Casado L, Moura L, Crespo C, Malta DC. Exposição ao tabagismo entre escolares no Brasil. Ciênc. Saúde Coletiva. 2010; 15(2): 3027-34.

3. Bastos JP, Araújo CLP, Hallal PC. Prevalence of insufficient physical activity and associated factors in Brazilian adolescents. J Phys Activity Health. 2008; 5:777-94.

4. Fernandes RA, Christofaro DGD, Casonatto J, Kawaguti SS, Ronque ERV, Cardoso $\mathrm{JR}$, et al. Associação transversal entre hábitos alimentares saudáveis e não saudáveis e atividade física de lazer em adolescentes. J Pediatr. 2011;87(3): 252-6.

5. Malcon MC, Menezes AMB, Maia, MFS; Chatkin M, Victora, CG. Prevalência e fatores de risco para tabagismo em adolescentes na América do Sul: uma revisão sistemática da literatura. Rev Panam Salud Publica. 2003; 13(4):222-228.

6. Hallal PC, Knuth AG, Cruz DKA, Mendes MI, Malta DC. Prática de atividade física em adolescentes brasileiros. Ciênc. Saúde Coletiva. 2010; 15(2): 3035-42.

7. Martín-Matillas M, Ortega FB, Chillon P, Pérez IJ, Ruiz JR, Castillo R, et al. Physical activity among Spanish adolescents: Relationship with their relatives' physical activity - The AVENA Study. J Sports Sci. 2011; 29 (4): 329-36.

8. Timperio AF, Stralen MMV, Brug J, Bere E, Chinapaw MJM, Bourdeaudhuij ID, et al. Direct and indirect associations between the family physical activity environment and sports participation among 10-12 year-old European children: testing the EnRG framework in the ENERGY project. Int J Behav Nutr Phys Act. 2013; 10-5.

9. Buist, KL; Dekovic, M; Prinzie, P. Sibling relationship quality and psychopathology of children and adolescentes: a meta-analysis. Clin Psychol Rev. 2013; 33(1): 97-106.

10. Instituto Brasileiro de Geografia e Estatística (Ibge). Censo Demográfico de 2010. Rio de Janeiro: Fundação Instituto Brasileiro de Geografia e Estatística. Ibge, 2010.

11. Instituto Nacional de Pesquisas e Estudos Anísio Teixeira (Inep). Censo Escolar 2009. Brasília: Ministério da Educação. 2012.

12. Lohman TG, Roche AF, Martorell R. Anthropometric Standardisation Reference Manual. Champaign, IL: Human Kinetics Books. 1988.

13. Must A, Dallal GE, Dietz WH. Reference data for obesity: 85th and 95th percentiles of body mass index (wt/ht2) and triceps skinfold thickness. Am J Clin Nutr. 1991; 53:839-46.

14. Biddle S, Cavill N, Sallis J. Young and active? Young people and health-enhancing physical activity-evidence and implications. Health Education Authority; 1998.

15. Carlini EA, Galduroz JCF, Noto AR, Nappo SA. I Levantamento domiciliar sobre drogas psicotrópicas no Brasil. Estudo envolvendo as 107 maiores cidades do país-2001. São Paulo: CEBRID. Centro Brasileiro de Informações sobre Drogas Psicotrópicas: UNIFESP. Universidade Federal de São Paulo; 2002.

16. Jose, KA; Blizzard, L; Dwyer, T; McKercher, C; Venn, A. Childhood and adolescent predictors of leisure time physical activity during the transition from adolescence to adulthood: a population based cohort study. Int J Behav Nutr Phys Act. 2011; 8:54.

17. Seabra AF, Mendonça DM, Thomis MA, Anjos LA, Maia LA. Determinantes biológicos e sócio-culturais associados à prática de atividade física de adolescentes. Cad. Saúde Pública. 2008; 24(4): 721-36.

18. Hallal ALC, Gotlieb SLD, Almeida LM, Casado L. Prevalência e fatores associados ao tabagismo em escolares da região sul do Brasil. Rev. Saúde Públ. 2009; 43(5): 779-88.

19. Abreu MNS, Souza CFS, Caiaffa WT. Tabagismo entre adolescentes e adultos jovens de Belo Horizonte, Minas Gerais, Brasil: influência do entorno familiar e grupo social. Cad. Saúde Pública. 2011; 27(5): 935-43. 
20. Bahr SJ, Hoffmann JP, Yang X. Parental and Peer Influences on the Risk of Adolescent Drug Use. J Prim Prev. 2005; 26(6).

21. Tjora T, Hetland J, Aarø LE, Øverland S. Distal and proximal family predictors of adolescents' smoking initiation and development: a longitudinal latent curve model analysis. BMC Public Health. 2011; 9;11:911.

22. Sharma R, Grover VL, Chaturvedi S. Tobacco Use Among Adolescent Students and the Influence of Role Models. Indian J Community Med. 2010; 35(2): 272-5.

23. Vieira PC, Aerts DRGC, Freddo SL, Bittencourt A, Monteiro L. Uso de álcool, tabaco e outras drogas por adolescentes escolares em município do Sul do Brasil. Cad. Saúde Pública. 2008; 24 (11): 2487-98.

24. Andrade SSCA, Yokota RTC, Sá NNB, Silva MMA, Araújo WN, Mascarenhas MDM, et al. Relação entre violência física, consumo de álcool e outras drogas e bullying entre adolescentes escolares brasileiros. Cad. Saúde Pública. 2012; 28(9): 1725-36.

25. Strauch ES, Pinheiro RT, Silva RA, Horta BL. Uso de álcool por adolescentes: estudo de base populacional. Rev. Saúde Pública. 2009; 43(4): 647-55.

26. Friese B, Grube JW, Moore RS. How Parents of Adolescents Store and Monitor Alcohol in the Home. J Prim Prev. 2012; 33(2-3): 79-83.

27. Trim RS, Leuthe E, Chassin L: Sibling influence on alcohol use in a young adult, highrisk sample. J Stud Alcohol. 2006; 67:391-8.

28. Ruiz-Juan F, Ruiz-Risueño J. Variables predictoras de consumo de alcohol entre adolescentes españoles. An Psicologia. 2011; 27 (2): 350-9.

29. Instituto Brasileiro de Geografia e Estatística (Ibge). Pesquisa Nacional de Saúde do Escolar - PeNSE. Rio de Janeiro: 2009.

30. Al-Isa AN, Campbell J, Desapriya E. "Factors Associated with Overweight and Obesity among Kuwaiti Elementary Male School Children Aged 6-10 Years" Int J Pediatr. 2010; 1-6.

ENDERECTO PARA

CORRESPONDÊNCIA

CHIRLE DE OLIVEIRA RAPHAELLI

Universidade Federal de Pelotas.

Escola Superior de Educação Física.

R. Luís de Camões, 625 - Três Vendas.

Pelotas - RS, 96055-630. Telefone/fax: (053) 3273-2752.

E-mail: chirleraphaelliahotmail..com
RECEBIDO 16/11/2014

REVISADO 26/01/2015

APROVADO 12/02/2015 